\title{
GRADE EVIDENCE TO DECISION (ETD) FRAMEWORK FOR COVERAGE DECISIONS
}

Elena Parmelli

Department of Epidemiology, Lazio Regional Health Service, ASL Roma I, Italian Cochrane

Centre, Department of Diagnostic, Clinical and Public Health Medicine,

University of Modena and Reggio Emilia

e.parmelli@deplazio.it

Laura Amato

Department of Epidemiology, Lazio Regional Health Service, ASL Roma 1

Andrew D. Oxman

Centre for Informed Health Choices, Norwegian Institute of Public Health

Pablo Alonso-Coello

Iberoamerican Cochrane Center, IIB Sant Pau-CIBERESP; Health Research Methods, Evidence, and Impact (formerly "Clinical Epidemiology and Biostatistics") and of Medicine,

McMaster University

\section{Massimo Brunetti}

Local Health Authority, Modena
Jenny Moberg

Global Health Unit, Norwegian Knowledge Centre for the Health Services

Francesco Nonino

Drug and Devices Evaluation Area, Emilia-Romagna Region, Bologna

Silvia Pregno

Local Health Authority, Modena

Carlo Saitto

Local Health Authority ASL Roma 1, Rome

Holger J. Schünemann

Departments of Health Research Methods, Evidence, and Impact (formerly "Clinical

Epidemiology and Biostatistics") and of Medicine, McMaster University

Marina Davoli

Department of Epidemiology, Lazio Regional Health Service - ASL Roma 1

the GRADE Working Group

Objectives: Coverage decisions are decisions by third party payers about whether and how much to pay for technologies or services, and under what conditions. Given their complexity, a systematic and transparent approach is needed. The DECIDE (Developing and Evaluating Communication Strategies to Support Informed Decisions and Practice Based on Evidence) Project, a GRADE (Grading of Recommendations Assessment, Development and Evaluation) Working Group initiative funded by the European Union, has developed GRADE Evidence to Decision (EtD) framework for different types of decisions, including coverage ones.

Methods: We used an iterative approach, including brainstorming to generate ideas, consultation with stakeholders, user testing, and pilot testing of the framework.

Results: The general structure of the EtD includes formulation of the question, an assessment using twelve criteria, and conclusions. Criteria that are relevant for coverage decisions are similar to those for clinical recommendations from a population perspective. Important differences between the two include the decision-making processes, accountability, and the nature of the judgments that need to be made for some criteria. Although cost-effectiveness is a key consideration when making coverage decisions, it may not be the determining factor. Strength of recommendation is not directly linked to the type of coverage decisions, but when there are important uncertainties, it may be possible to cover an intervention for a subgroup, in the context of research, with price negotiation, or with restrictions.

Conclusions: The EtD provides a systematic and transparent approach for making coverage decisions. It helps ensure consideration of key criteria that determine whether a technology or service should be covered and that judgments are informed by the best available evidence.

Keywords: GRADE, Coverage decisions

Members of the GRADE Working Group who contributed to writing this study include: Elena Parmelli, Laura Amato, Andrew D Oxman, Pablo Alonso-Coello, Massimo Brunetti, Jenny Moberg, Francesco Nonino, Silvia Pregno, Carlo Saitto, Holger J Schünemann, Marina Davoli, Elie A Akl, Roberto D’Amico, Philippe Duclos, Nicola Magrini, Joerg J Meerpohl, Lorenzo Moja, Reem Mustafa, Daria 0'Reilly, Sarah Rosenbaum, Melanie Schuster, Jasvinder Singh, Jean-Eric Tarride. We thank Roman Jaeschke and Zuzanna Mitrova for their comments and suggestions. Author Contributions: E.P., L.A., A.D.O., M.D. wrote the first draft of this manuscript and E.P., L.A., A.D.O., P.A.C., M.B., J.M., F.N., S.P., C.S., H.J.S., M.D., E.A.A., R.D., P.D., N.M., J.J.M., L.M., R.M., D.O., S.R., M.S., J.S., J.E.T. contributed to the writing. All authors have read and commented on the results and conclusions in the manuscript, and have given written agreement of their authorship. Work on this article has been partially funded by the European Commission FP7 Program (grant agreement 258583) as part of the DECIDE project. Sole responsibility lies with the authors; the European Commission is not responsible for any use that may be made of the information contained therein. E.P. and L.M. are recipients of a Research Early Career Award
Coverage decisions are decisions by third party payers (public or private health insurers) about whether and how much to pay for drugs, tests, devices, or services and under what conditions. These decisions are made at national, regional, or local levels, depending on the type of interventions and the way health services are paid for in a country. Often, committees or panels that may include policy makers, managers, clinicians,

from the Italian Ministry of Health (GR-2011-02348048). P.A.C. is supported by a Miguel Servet investigator contract from the Instituto de Salud Carlos III (CPII15/0034). S.J. is supported by grants from the Agency for Health Quality and Research Center for Education and Research on Therapeutics (CERTs), National Institute of Arthritis, Musculoskeletal and Skin Diseases (NIAMS), National Institute of Aging (NIA) and National Cancer Institute (NCI) and by the resources and use of facilities at the Birmingham VA Medical Center, Alabama, USA. 
and researchers, make these decisions. Processes and criteria used for coverage decisions vary across and within countries $(1 ; 2)$. Processes for deciding which drugs to cover have become increasingly systematic and evidence-based in many countries and often include economic evaluations. Processes for making coverage decisions for other technologies and services in health care vary much more. Often coverage decisions focus on new technologies and services (interventions). However, increasing attention is being given to decisions about whether to stop coverage for interventions that are not effective or not cost-effective (3), as in the scenario in Table 1. A decision not to cover an intervention can make resources available for other interventions that are effective or more cost-effective.

Like other healthcare decisions, coverage decisions require consideration of multiple factors $(1 ; 2 ; 4 ; 5)$. Concerns about costs, effectiveness and cost-effectiveness of health interventions have dominated the debate in a wide range of countries for a long time (6). More recently, the use of equity-related criteria, such as socio-economic status and gender, have been put forward (7). Other criteria, such as ease of implementation and political acceptability, are also sometimes considered in the prioritization of health interventions (6).

Conflicting interests, particularly financial interests, can affect coverage decisions in undesirable ways (3). For example, manufacturers want to ensure coverage to make a profit on their investment and are likely to lobby for coverage of their products (sometimes using clinicians or patient groups). Health insurance companies making decisions want to contain costs to ensure their profits, but may also want to ensure coverage in order not to lose members, and politicians may want to avoid antagonizing voters or lobbyists (8).

Coverage decisions need to be fair. This requires that they are relevant, transparent, possible to revise, and documented (9). A systematic and transparent approach based on the best available evidence can help to structure a well-informed and accountable decision-making processes, and to reduce potential disagreements about coverage decisions. The GRADE (Grading of Recommendations Assessment, Development and Evaluation) Evidence to Decision (EtD) framework for coverage decisions can help to ensure that decision-making processes adhere to these principles.

Coverage decisions and clinical or public health recommendations share some common features. Both require formulation of a question, an assessment, and conclusions. Formulating the question requires similar considerations. However, whereas guideline panels can make clinical recommendations from the perspective of an individual patient, coverage decisions are always made from a population perspective (10). The criteria that affect a decision are similar, but there are some important differences in relation to panels' judgments about how much people value the main outcomes, equity, acceptability, and feasibility.
Although coverage decisions are always made from a population perspective, they have clinical implications for individual patients. For example, if a decision is made to discontinue coverage of opportunistic prostate cancer screening for asymptomatic men (Table 1), it is likely that fewer men will choose to be screened because of the out-of-pocket cost of the test.

The development of the EtD frameworks and their rationale for different types of decisions are described elsewhere (11). In this article, we describe the use of EtD frameworks for coverage decisions. We will illustrate how they can help those responsible for making coverage decisions and the people affected by those decisions. A glossary of terminology used in EtD frameworks is available in Supplementary File 1.

\section{METHODS}

The EtD framework for coverage decisions has been developed as part of the DECIDE (Developing and Evaluating Communication Strategies to Support Informed Decisions and Practice Based on Evidence) Project, using an iterative process as previously described $(11 ; 12)$. The initial development began with the GRADE Working Group's approach to clinical recommendations and included iterative use of brainstorming workshops to generate ideas and potential solutions, consultation with an advisory group, user testing, and feedback to inform revisions from a user perspective, and application of the framework to different types of coverage decisions.

\section{RESULTS}

The structure of the EtD framework for coverage decisions is the same as for other EtD frameworks $(10 ; 11 ; 13)$. It includes the question, an assessment, and conclusions. The criteria are similar to those in the EtD framework for clinical decisions from a population perspective (10). In this article, we focus on elements of the EtD framework that are of particular importance for coverage decisions and provide examples. Supplementary File 2 is an example an EtD framework for the scenario in Table 1. It was prepared by a technical team including epidemiologists, an economist and a decision maker; the judgements reported are those of the technical team.

\section{Formulating the Question}

In the scenario described in Table 1, the question posed was "Should we stop covering opportunistic screening for prostate cancer in asymptomatic men?" Question details include the population (asymptomatic men aged $>50$ ), intervention (opportunistic screening), comparison (no screening), outcomes (all-cause mortality, cause specific mortality, number of men diagnosed with prostate cancer, harms), setting (Italy), and the perspective (that of a Regional Health Authority) (Table 2). In this context, taking the perspective of the Regional Health Authority means only taking account of the NHS costs. The 
Table 1. Scenario: Should We Stop Covering Opportunistic Screening for Prostate Cancer in Asymptomatic Men?

Prostate cancer screening using prostate-specific antigen (PSA) has previously been widely recommended and widely used in Italy. However, the results of randomized trials have found that screening has small, if any effects on all-cause mortality and prostate cancer mortality, and recent clinical practice guidelines have recommended against screening (21).

In Italy, Regional Health Authorities make decisions about payment for interventions (coverage) within the National Health Service. Typically, decisions about coverage have focused on new interventions and this continues to be an important consideration. However, given the economic situation and the need to contain costs within the Health Service, discontinuing coverage of interventions that are not effective or cost effective (disinvestment) has become an important consideration for Regional Health Authorities. In this context, a Regional Health Authority asked the question: Should we stop paying for PSA testing when it is used to screen asymptomatic men for prostate cancer?

Table 2. Evidence to Decision (EtD) Framework: Question Section

Should we stop covering opportunistic screening for prostate cance
Patients: Asymptomatic men over 50 years
Intervention: Opportunistic screening with prostate specific
antigen (PSA)
Comparison: No screening
Main outcomes: All-cause mortality, prostate cancer mortality,
quality of life, harms
Setting: The National Health Service in ltaly
Perspective: Regional Health Authority

Note. Templates used for EtD frameworks are adapted for specific types of decisions. The one shown here is for a coverage decision. It is for a hypothetical decision. An electronic version is available at http://ietd2.epistemonikos.org/\#/list/examples.

specific costs that were considered are specified in the section on resource use.

Although coverage decisions always take a population perspective, the perspective of interest should be specified. For example, a decision about national coverage might take a societal perspective (considering all costs, regardless of who pays or saves), a government perspective or the perspective of the health department or the National Health Service (only considering costs and savings to the government or a specific budget).

\section{Making an Assessment}

Assessments in EtD frameworks for coverage decisions address twelve factors (criteria) that can influence coverage decisions (Table 3). For each criterion, decision makers must make a judgement informed by the best available evidence (6).

Judgments about how much of a priority a problem is, how substantial the effects are, how large the costs are, and how cost-effective an intervention is require a comparison. Organizations making coverage decisions can use explicit or implicit thresholds or standards for making these judgments. Some organizations, for example, have explicit thresholds for cost-effectiveness $(6 ; 14)$. They might also have thresholds or standards for other criteria. For example, the Australian Pharmaceutical Benefits Scheme (PBS) is influenced by whether the
Table 3. Criteria of the EtD Framework for Coverage Decisions

- Is the problem a priority?

- How substantial are the desirable anticipated effects?

- How substantial are the undesirable anticipated effects?

- What is the overall certainty of the evidence of effects?

- Is there important uncertainty about in how much people value the main outcomes?

- Does the balance between desirable effects and undesirable effects favour the option or the comparison?

- How large are the resource requirements (costs)?

- What is the certainty of the evidence of resource use?

- Does the cost-effectiveness of the option favour the option or the comparison?

- What would be the impact on health equity?

- Is the option acceptable to key stakeholders?

- Is the option feasible to implement?

disease being treated is life-threatening ( $<5$ year expected survival) (6) This could be used as an explicit threshold about how much of a priority a problem is.

While thresholds might improve consistency and transparency, they are somewhat arbitrary and problematic $(15 ; 16)$. Moreover, because there are multiple factors that determine a 
decision and those factors may interact, the use of thresholds may be inappropriate. For example, an analysis of decisions about public insurance based on data from the PBS found no evidence of a fixed threshold for cost-effectiveness (6). Coverage decisions were related to the characteristics of the clinical condition, confidence in the evidence of effectiveness and total cost to the government, as well as cost-effectiveness.

\section{Priority of the Problem}

Coverage decisions are often influenced by whether the problem being treated is a priority. For example, treatments for minor illnesses are often not covered, treatments for severe illnesses are sometimes prioritized for coverage, and political policies sometimes set priorities for coverage decisions based on perceptions of how important certain types of problems are.

How common a condition is, that is how many people have it, also can influence decisions, but not independently. A government or organization making coverage decisions might have a policy of prioritizing conditions that are responsible for a large burden of disease. In this case, it is the severity of the condition together with how common it is that determine the burden of disease. Other governments or organizations making coverage decisions might have a policy of prioritizing rare conditions.

Both of those policies have consequences for what is covered. The first policy discriminates for people with common conditions and against people with rare conditions. The second policy does the opposite. Both are reasonable, but some people are likely to consider either one unfair. Therefore, it is important that organizations making coverage decisions are transparent and consistent about which, if either of those policies affects whether an intervention is covered or not.

In the absence of one of those two policies, the number of people who have a condition is not directly relevant to whether a problem is a priority. The number of people with a condition can affect a coverage decision in other ways, but only because of how that affects one of the other criteria. For example, the resources required (costs) for an intervention are determined in part by the number of people with a condition (the seventh criterion in Table 3) and the number of people with a condition can affect a health system's capacity to treat people and consequently the feasibility of implementing a coverage decision (the last criterion in Table 3).

People making a coverage decision might consider a problem a priority because it is already costing a lot of money and they are considering covering an alternative intervention that will save money or they are considering stopping payment for an intervention that costs a lot of money and has marginal benefits, as was the case in the scenario in Table 1. In that case, they should be clear that it is the potential savings that makes a problem a priority. The potential savings is affected by how common the condition is, but it is not the number of people with the condition per se that is relevant. In the scenario, stopping coverage of opportunistic screening for prostate cancer, the judgement that this probably is a priority was influenced by the potential savings, and consequently the number of people affected.

\section{Certainty of the Evidence of Effects}

Low certainty of the evidence of effects can affect coverage decisions, for example, if the desirable effects are uncertain, that may drive a decision not to cover an intervention. If an intervention is promising, but there is important uncertainty, depending on the degree of uncertainty and other factors, a decision may be taken to cover it with monitoring of potential adverse effects, to cover it only in the context of research $(17 ; 18)$, or not to cover it until there is more evidence.

In most cases, the main outcomes that are relevant for coverage decisions are those that people who use an intervention might experience. However, occasionally outcomes experienced by other people might be important for coverage decisions. For example, for coverage decisions about antibiotics, the development of antibiotic resistance might be an important outcome.

In the scenario in Table 1, evidence of the effects of screening for prostate cancer came from a Cochrane systematic review (19). The review found that screening has little or no effect on all-cause mortality and cancer-specific mortality, with moderate certainty of the evidence from randomized trials (see definition in Supplementary File 1). Localized prostate cancer was more commonly diagnosed and advanced prostate cancer was less commonly diagnosed in men randomized to screening compared with the men serving as controls. However, there is evidence that screening does not improve quality of life and it caused a range of minor to major harms (20). Common minor harms included bleeding, bruising, and short-term anxiety. Common major harms included, erectile dysfunction, incontinence, infections, blood loss requiring transfusion, pneumonia and overdiagnosis and overtreatment, which may be responsible for additional harms.

Modeling is necessary to estimate the magnitude of these effects (13) and the certainty of the evidence is low or very low for some of these effects. Detailed judgements underlying the assessment of the certainty of the evidence are shown in the "Evidence Profile" at the end of the EtD framework in Supplementary File 2. Given that there is no evidence of benefits, this uncertainty was not important for an overall decision about coverage. It could become relevant, if decision makers concluded that there was adequate evidence to support screening for a subgroup of men, such as men between 55 and 69. The largest trial of screening included in this systematic review found a small reduction in prostate cancer deaths in that subgroup ( 1 of 1,000 men screened over 11 years), but the certainty of the evidence for that subgroup effect was low or very low (21). 


\section{Uncertainty about How Much the People Affected Value the Main Outcomes}

How much the people affected by the intervention value, on average, the main outcomes plays an important role in deciding whether to cover an intervention, similarly to clinical recommendations (10). By important uncertainty, we mean that we are unsure about how much value people place on the main outcomes, including how important they are relative to each other.

For example, chemotherapy for cancer usually has serious adverse effects. Deciding whether the desirable effects of chemotherapy outweigh the undesirable effects requires a judgment about how important the desirable outcomes are (e.g., increased survival) compared with the undesirable outcomes (e.g., nausea, hair loss, increased risk of infections).

A similar situation is described in the PSA example (Supplementary File 2) where, according to the research evidence presented (22) men were willing to trade-off some risk reduction of prostate cancer related death to be relieved of the burden of biopsies or unnecessary treatments.

A panel may decide to postpone a decision or to cover an intervention in the context of research if there is important uncertainty about how much people value the main outcomes.

Unlike for clinical recommendations, variability in how much people value the main outcomes is not relevant for coverage decisions: as long as an important proportion of people would value the desirable effects more than the undesirable effects, covering the intervention for them might be justified and others could choose not to use the intervention.

\section{Resource Use}

Economic evaluations play an important role in coverage decisions, because obtaining value for money is central to these decisions. The greater the costs of an intervention and the less cost-effective it is, the less likely it is that it should be covered (7). Important uncertainty about the incremental costeffectiveness of an intervention compared with standard care might be a reason to postpone a decision until better evidence is available or to cover the intervention with monitoring of effects and expenditures.

Some countries require an economic evaluation as part of the submission requirements for coverage decisions, and most of them also require a budget impact analysis as part of the reimbursement dossier (23). There are examples of institutions using largely cost-effectiveness information to drive their decisions: for example, cost-effectiveness predicted 82 percent of coverage decisions at National Institute for Health and Care Excellence (NICE) (24). Many organizations making coverage decisions have limited, if any resources to conduct economic evaluations. They often rely on manufacturers' submissions or published economic evaluations, which are often poorly done and reported, and may not be applicable (25). There may also be political restrictions or market forces that limit the ability of panels making coverage decisions to use economic evaluations (26). Because resource use is always relevant to coverage decisions (implicitly, if not explicitly), panels should be as systematic and transparent as possible about how they considered resource use, and about any assumptions that they have made.

For the scenario in Table 1, although the cost of PSA screening for individual patients was small ( $€ 7.4$ per test), savings on the test alone could be as much as $€ 400,000$ per year for the Local Health Authority if opportunistic screening was no longer covered (Supplementary File 1). In addition, there would be savings from the costs of unnecessary biopsies for false positive PSA tests and less unnecessary treatment (see Supplementary File 2).

\section{Equity}

Equitable access to interventions is an important consideration for coverage decisions. Not covering an intervention for which the desirable effects outweigh the undesirable effects means that people who cannot afford the intervention will be denied access to it thereby increasing inequity. Conversely, covering an intervention can reduce inequities.

In the scenario in Table 1, it was assessed that stopping coverage would probably not have an impact on equity.

\section{Acceptability}

Acceptability considerations are similar for clinical recommendations and coverage decisions and includes consideration of the distribution of the benefits, harms, and costs, as well as ethical considerations (10). It refers to acceptability of the interventions by stakeholders affected by coverage decisions. Those stakeholders include people who might directly or indirectly benefit from coverage, people who would not benefit, but would share the cost of the intervention (through premiums or taxes), health professionals, producers of the health technologies, and organizations that represent these and other groups of people with an interest in the intervention.

Deciding not to cover an intervention because of costs or cost-effectiveness might be unacceptable to patients who would benefit from the intervention, and to clinicians who care for those patients. Conversely, deciding to cover expensive interventions might not be acceptable to those who would not benefit, but would share the costs of the intervention. While these considerations typically should not change a decision about whether to cover an intervention, they should be taken into account when considering dissemination and implementation of the decision. Similar considerations might apply to a decision to restrict coverage, if some people who would be denied coverage could benefit from the intervention.

In the prostate screening example, PSA screening was widely used in men over 50 . Stopping coverage for this intervention could lead to problems of acceptability for men who want to be screened because they previously had been screened, 
because they have a family history of prostate cancer, or for other reasons. Nonetheless, the technical team judged that there probably would not be important problems with the acceptability of stopping coverage, provided the justification for the decision was communicated clearly.

\section{Feasibility}

Feasibility considerations for coverage decisions are different from feasibility considerations for clinical recommendations. For coverage, feasibility considerations focus primarily on the feasibility of implementing coverage, restricted coverage or no coverage for an intervention. A feasibility issue may arise from the difficulty of implementing an effective administrative procedure to ensure that coverage is limited to a specific population of patients identified by clinical characteristics. For example, this might be an important consideration for a decision to restrict coverage of PSA screening to men with a family history of prostate cancer.

Capacity to meet increased demand for an intervention can also be an important consideration. For example, a health system's capacity to provide colonoscopy might be an important consideration for a decision about whether to cover screening colonoscopy for colon cancer. Similar to considerations about acceptability, these considerations might not change a decision about whether to cover an intervention, but should be taken into account when considering dissemination and implementation of the decision.

\section{Drawing Conclusions}

The EtD framework for coverage decisions includes five options for coverage decisions: no coverage, coverage with evidence development (in the context of research), coverage with price negotiation, restricted coverage, and full coverage. Price negotiation may include risk-sharing agreements between manufacturers and payers (27). One or more of the criteria used to assess interventions can drive decisions about coverage, and this can vary from decision to decision. For example, a decision about coverage for opportunistic prostate cancer screening might be driven by the evidence that there are small, if any benefits and there are important harms.

A decision to cover an intervention in the context of research can be made when there is important uncertainty about the effects of an intervention. Although this is an attractive option for new, promising interventions, it has been difficult to implement $(17 ; 18)$. Coverage with price negotiation is common for new, effective drugs that do not meet standards for resource use or cost effectiveness. Restricted coverage is also commonly used for interventions that are only beneficial or cost-effective for a subgroup of patients. Restricted coverage is similar to a conditional recommendation that specifies a subgroup of patients for whom a technology is recommended.
Deciding to cover an intervention is not the same as recommending it (10). It is compatible with either a strong clinical recommendation (meaning that most people would want the intervention and only a small proportion would not) or a weak clinical recommendation (meaning that the majority of people would want the intervention, but many would not).

For coverage decisions, it may be particularly important to monitor usage, inappropriate usage and costs of the intervention for which coverage is being provided. When inappropriate use is a concern, it may be possible to monitor this by using registries or other routinely collected data. However, this requires that reliable data are collected and that observed figures can be compared with prespecified optimal standards, preferably agreed with the stakeholders affected by coverage decisions. This will help distinguishing between appropriate and inappropriate use.

In the scenario in Table 1, the decision was to stop covering opportunistic PSA screening for asymptomatic men (Supplementary File 2). This decision was driven by an assessment of there being no important benefits in terms of mortality or quality of life, and there being several undesirable effects.

\section{DISCUSSION}

The main strengths of the EtD framework for coverage decisions are its design and structure, summarizing in a logical and transparent way all the elements of a complex decision-making process. The EtD framework guides consideration of the important factors that should determine a decision about coverage, and can help in avoiding potential inappropriate influences (e.g., lobbying by manufacturers). The application of a structured, explicit and transparent approach to coverage decisions was perceived as a strong point in favor of using the EtD framework, and its innovative nature was particularly appreciated by participants in user testing and pilot tests $(11 ; 28)$.

From the perspective of clinicians, patients, and the public, use of the EtD framework can help to ensure that decisions are fair. It documents and helps to ensure consistent use of appropriate criteria for assessing interventions and transparent use of evidence to inform judgements for each criterion. It can facilitate identification of reasons for disagreements and feedback on a draft decision before making a final one.

The main weakness is the usability of the framework by stakeholders with different levels of methodological knowledge. However, it might also be considered a potentially useful instrument to facilitate better understanding of the methodological considerations that are inherent in evidence-informed coverage decisions.

The criteria that are used to assess interventions in the EtD framework for coverage decisions are not new. They are similar to criteria already used by many organizations, and to the criteria suggested by the GRADE Working Group for clinical 
recommendations $(10 ; 11 ; 13)$. However, other frameworks frequently fail to consider key factors and do not transparently use evidence to inform judgements about each key factor. For example, an overview of existing frameworks to support coverage decisions for vaccines highlighted differences in the taxonomy used by different frameworks, and that key factors were not consistently considered (29). The EtD frameworks provides a way for organizations to monitor their decisions, and they can facilitate sharing, comparing and learning across organizations that make coverage decisions.

\section{SUPPLEMENTARY MATERIAL}

Supplementary File 1:

https://doi.org/10.1017/S0266462317000447

Supplementary File 2:

https://doi.org/10.1017/S0266462317000447

\section{CONFLICTS OF INTEREST}

Authors are members of the GRADE Working Group and the DECIDE project.

SJ has no financial or non-financial conflicts related directly to this study; he has received research and travel grants from Takeda and Savient; and consultant fees from Savient, Takeda, Allergan, and Regeneron.

\section{REFERENCES}

1. Fischer KE. A systematic review of coverage decision-making on health technologies-evidence from the real world. Health Policy. 2012;107:218-230.

2. Guindo LA, Wagner M, Baltussen R, et al. From efficacy to equity: Literature review of decision criteria for resource allocation and healthcare decisionmaking. Cost Eff Resour Alloc. 2012;10:9.

3. Henshall C, Schuller T, Mardhani-Bayne L. Using health technology assessment to support optimal use of technologies in current practice: The challenge of "disinvestment". Int J Technol Assess Health Care. 2012;28:203-210.

4. Tromp N, Baltussen R. Mapping of multiple criteria for priority setting of health interventions: An aid for decision makers. BMC Health Serv Res. 2012;12:454.

5. Tony M, Wagner M, Khoury H, et al. Bridging health technology assessment (HTA) with multicriteria decision analyses (MCDA): Field testing of the EVIDEM framework for coverage decisions by a public payer in Canada. BMC Health Serv Res. 2011;11:329.

6. Harris AH, Hill SR, Chin G, Li JJ, Walkom E. The role of value for money in public insurance coverage decisions for drugs in Australia: A retrospective analysis 1994-2004. Med Decis Making. 2008;28: 713-722.

7. Ottersen TNO, Chitah BM, Cookson R, Daniels N, et al. Making fair choices on the path to universal health coverage. Final report of the WHO Consultative Group on Equity and Universal Health Coverage. Geneva: WHO; 2014.

8. Faunce TA. Challenges for Australia's bio/nanopharma policies: Trade deals, public goods and reference pricing in sustainable industrial renewal. Aust New Zealand Health Policy. 2007;4:9.

9. Daniels N. Accountability for reasonableness. BMJ. 2000;321:13001301.
10. Alonso-Coello P, Oxman AD, Moberg J, et al. GRADE Evidence to Decision (EtD) frameworks: A systematic and transparent approach to making well informed healthcare choices. 2: Clinical practice guidelines. BMJ. 2016;353:i2089.

11. Alonso-Coello P, Schünemann HJ, Moberg J, et al. GRADE Evidence to Decision (EtD) frameworks: A systematic and transparent approach to making well informed healthcare choices. 1: Introduction. BMJ. 2016;353:i2016.

12. Treweek S, Oxman AD, Alderson P, et al. Developing and evaluating communication strategies to support informed decisions and practice based on evidence (DECIDE): Protocol and preliminary results. Implement Sci. 2013;8:6.

13. Schünemann HJ, Mustafa R, Brozek J, et al. GRADE Guidelines: 16. GRADE evidence to decision frameworks for tests in clinical practice and public health. J Clin Epidemiol. 2016;76:89-98.

14. Claxton K, Martin S, Soares M, et al. Methods for the estimation of the National Institute for Health and Care Excellence cost effectiveness threshold. Health Technol Assess. 2013;19:1-503.

15. Rocchi A, Menon D, Verma S, Miller E. The role of economic evidence in Canadian oncology reimbursement decision-making: To lambda and beyond. Value Health. 2008;11:771-783.

16. Drummond MF, Schwartz JS, Jonsson B, et al. Key principles for the improved conduct of health technology assessments for resource allocation decisions. Int J Technol Assess Health Care. 2008;24:244-258; discussion 362-368.

17. Levin L, Goeree R, Levine M, et al. Coverage with evidence development: The Ontario experience. Int J Technol Assess Health Care. 2011;27:159-168.

18. Hutton J, Trueman P, Henshall C. Coverage with evidence development: An examination of conceptual and policy issues. Int J Technol Assess Health Care. 2007;23:425-432.

19. Ilic D, Neuberger MM, Djulbegovic M, Dahm P. Screening for prostate cancer. Cochrane Database Syst Rev. 2013;1:CD004720.

20. Heijnsdijk EA, Wever EM, Auvinen A, et al. Quality-of-life effects of prostate-specific antigen screening. N Engl J Med. 367;367:595-605.

21. Djulbegovic M, Beyth RJ, Neuberger MM, et al. Screening for prostate cancer: Systematic review and meta-analysis of randomised controlled trials. BMJ. 2010;341:c4543.

22. de Bekker-Grob EW, Rose JM, Donkers B, et al. Men's preferences for prostate cancer screening: A discrete choice experiment. Br J Cancer. 2013; 108:533-541.

23. Drummond M. Twenty years of using economic evaluations for drug reimbursement decisions: What has been achieved? J Health Polit Policy Law. 2013;38:1081-1102.

24. Dakin H, Devlin N, Feng Y, Rice N, O’Neill P, Parkin D. The influence of cost-effectiveness and other factors on nice decisions. Health Econ. 2014 [Epub ahead of print].

25. Jefferson T, Demicheli V. Quality of economic evaluations in health care. BMJ. 2002;324:313-314.

26. Brunetti M, Shemilt I, Pregno S, et al. GRADE guidelines: 10. Considering resource use and rating the quality of economic evidence. $J$ Clin Epidemiol. 2013;66:140-150.

27. Garrison LP Jr, Towse A, Briggs A, et al. Performance-based risksharing arrangements-good practices for design, implementation, and evaluation: Report of the ISPOR good practices for performance-based risk-sharing arrangements task force. Value Health. 2013;16:703-719.

28. Neumann I, Brignardello-Petersen R, Wiercioch W, et al. The GRADE evidence-to-decision framework: A report of its testing and application in 15 international guideline panels. Implement Sci. 2016;11:93.

29. Gonzalez-Lorenzo M, Piatti A, Coppola L, et al. Conceptual frameworks and key dimensions to support coverage decisions for vaccines. Vaccine. 2015;33:1206-1217. 